\title{
Investigation of the clinicopathological features of fallopian tube malignancy
}

\author{
YOSHIHITO YOKOYAMA ${ }^{1}$, MASAYUKI FUTAGAMI ${ }^{1}$, TOSHIO FUJIMOTO $^{2}$, YUKIHIRO TERADA $^{2}$, \\ ERIKO TAKATORI $^{3}$, TORU SUGIYAMA ${ }^{3}$, TAKEO OTSUKI ${ }^{4}$, NOBUO YAEGASHI ${ }^{4}$, TAKANOBU KOJIMAHARA ${ }^{5}$, \\ HIROHISA KURACHI $^{5}$, HIROSHI NISHIYAMA ${ }^{6}$, KEIYA FUJIMORI ${ }^{6}$, TORU TASE $^{7}$ and HIDEKI MIZUNUMA $^{1}$ \\ ${ }^{1}$ Department of Obstetrics and Gynecology, Hirosaki University Graduate School of Medicine, Hirosaki 036-8562; \\ ${ }^{2}$ Department of Obstetrics and Gynecology, Akita University School of Medicine, Akita 010-8543; \\ ${ }^{3}$ Department of Obstetrics and Gynecology, Iwate Medical University School of Medicine, Morioka 020-8505; \\ ${ }^{4}$ Department of Obstetrics and Gynecology, Tohoku University School of Medicine, Aoba-ku, Sendai 980-8574; \\ ${ }^{5}$ Department of Obstetrics and Gynecology, Yamagata University School of Medicine, Yamagata 990-9585; \\ ${ }^{6}$ Department of Obstetrics and Gynecology, School of Medicine, Fukushima Medical University, \\ Fukushima 960-1295; 7 Department of Gynecology, Miyagi Cancer Center, Natori 981-1293, Japan
}

Received February 11, 2013; Accepted April 5, 2013

DOI: $10.3892 /$ or.2013.2439

\begin{abstract}
The present study investigated the clinicopathological features of fallopian tube malignancy (FTM) and elucidated the biological behavior of this disorder. Data were compiled concerning FTM from 68 patients from 7 institutes. The patients included 60 cases with fallopian tube carcinoma and 8 cases with fallopian tube carcinosarcoma. The clinical stage was stage III or higher in $72 \%$ of the cases. A complete response or partial response was achieved in 56 and 10 of the 68 patients with FTM, respectively, indicating a response rate of $97.1 \%$. The median observation period for FTM was 41 months (3 to 126 months). Three of the 19 patients with stage I/II disease $(16 \%)$ and 31 of the 49 patients with stage III/IV disease $(63 \%)$ experienced recurrence, with a median progression-free survival of 17.5 months, and a 3 -year overall survival of $77.2 \%$. Regarding the site of recurrence, local intraperitoneal recurrence $(26.2 \%)$ and solitary recurrences in lymph nodes $(19.0 \%)$ and in the liver (16.7\%) were relatively frequent. Secondary debulking surgery (SDS) was performed in 15 patients (44\%) out of the 34 recurrent FTMs Conversely, recurrence was associated with ascites (carcinomatous peritonitis) in 4 of the 34 recurrent patients, but all 4 patients died. The median survival period after recurrence was 28 months: 7.5 and 30 months with and without ascites,
\end{abstract}

Correspondence to: Dr Yosihihito Yokoyama, Department of Obstetrics and Gynecology, Hirosaki University Graduate School of Medicine, 5-Zaifu-cho, Hirosaki, Aomori 036-8562, Japan

E-mail: yokoyama@cc.hirosaki-u.ac.jp

Key words: primary carcinoma of the fallopian tube, primary carcinosarcoma of the fallopian tube, solitary recurrence, secondary debulking surgery, prognosis respectively $(\mathrm{P}<0.001)$. A univariate analysis showed that prognosis was significantly correlated only with whether SDS could be performed. These results suggest that since FTM frequently results in solitary recurrence, aggressive recurrence treatment including SDS could improve prognosis.

\section{Introduction}

Fallopian tube malignancy (FTM) is a rare disease that comprises only 0.14 to $1.8 \%$ of female genital malignancies (1). The incidence rate in the United States is estimated to be on average 3.6/million/year (2). Although rare, FTM is a disease that has increased 4.5 -fold over the past 40 years (3). Even though the staging and therapeutic strategy for fallopian tube carcinoma are based on those of ovarian carcinoma, it is difficult to conduct a large-scale clinical study exclusively on fallopian tube carcinoma because of its rarity, and the pattern of metastasis as well as time to recurrence are not clear. Wethington et al (4) compared the outcome of fallopian tube carcinoma and ovarian carcinoma based on the Surveillance, Epidemiology, and End Results (SEER) database of the National Cancer Institute, and showed that fallopian tube carcinoma was associated with a more favorable long-term outcome than ovarian carcinoma. However, the biological behaviors of fallopian tube carcinoma such as location of recurrence and time to recurrence remain unknown (4).

This study was a retrospective, multicenter study that aimed to investigate the clinicopathological features of FTM and to elucidate the biological behaviors of this disease.

\section{Materials and methods}

All patients with FTM treated between January 2001 and December 2011 were eligible for this study. Institutional review board approval was obtained at each of the 7 participating academic centers prior to data acquisition, and informed 
consent was obtained from the patients or the guardians. Cases were defined as FTM using diagnostic criteria as documented by Sedlis (5). Briefly, these criteria were as follows: i) the main tumor arises from the endosalpinx; ii) the histological pattern reproduces the endothelium of the tube mucosa; iii) transition from benign to malignant tubal epithelium is demonstrable; iv) the ovaries or endometrium are either normal or contain a tumor smaller than the tumor in the tube. In addition, we also adopted the definition of fallopian tube carcinoma of WHO classification which states that there is a primary lesion in the lumen or fimbriae of the fallopian tube and a lesion does not exist in the ovary and the uterine or it is different from the fallopian tube clearly even if it exists (6). All slides were reviewed by expert pathologists from each institution. A common database form was designed to be utilized by all participating centers. The medical records included information regarding age, gravidity and parity, clinical presentation, disease stage, type of surgery performed, treatment rendered, histological type, progression-free survival (PFS), overall survival (OS), and sites of recurrence. Patients of all stages were eligible. Staging was based on the International Federation for Obstetrics and Gynecology (FIGO) criteria. Procedures included in the standard surgery consisted of bilateral salpingo-oophorectomy, hysterectomy and greater omentectomy with or without cytoreduction. Staging laparotomy included retroperitoneal (pelvic, para-aortic) lymph node dissection (or biopsy) and intraperitoneal biopsies in addition to standard surgery. The $1-\mathrm{cm}$ cutoff was used as a threshold for optimal cytoreduction (7). When adjuvant chemotherapy was indicated, patients generally received platinum/taxan-based combination chemotherapy. Cases that received neoadjuvant chemotherapy also were included in this retrospective study.

Clinical response was assessed in the enrolled patients with lesions that could be measured according to the revised RECIST guideline (version 1.1) (8). A complete response (CR) was defined as the complete disappearance of all measurable lesions. A partial response (PR) was defined as a $30 \%$ or greater decrease in the sum of the measurable lesions. Stable disease was defined as a steady state of response less than PR or an increase in $<20 \%$ in the sum of the measurable lesions. Progressive disease was defined as an increase of $20 \%$ or more in the sum of the measurable lesions or the appearance of new lesions.

PFS was calculated from the date of the start of treatment to the date of recurrence or progression. OS was calculated from the date of the start of treatment to the date of death or last follow-up. The cumulative survival curve and median PFS time were estimated by use of the Kaplan-Meier method. Comparison between survival curves was carried out using the log-rank test. Difference in response rate between predictive variables was analyzed using the Chi-square test. Statistical significance was set at $\mathrm{P}<0.05$.

\section{Results}

Patient characteristics. Patient characteristics are shown in Table I. Sixty-eight patients with FTM were enrolled for this retrospective analysis. The 68 patients with this disease included 60 cases with fallopian tube carcinoma and 8 cases with fallopian tube carcinosarcoma. The median age of the patients

Table I. Patient characteristics.

Clinical factors

Fallopian tube malignancy

Carcinoma of the fallopian tube

Carcinosarcoma of the fallopian tube

Median age (range), in years

History of delivery

Yes

No

Menstruation

Premenopause

Postmenopause

Chief complaint

Lower abdominal pain or distension

Atypical genital bleeding

Feeling of abdominal mass

Bloody bowel discharge

Asymptomatic

Others

Serum CA-125, median (range), U/ml

Preoperative diagnosis

Ovarian carcinoma

Fallopian tube carcinoma

Endometrial carcinoma

Colon carcinoma

Others

Neoadjuvant chemotherapy

Yes

No

FIGO stage

I

II

III

IV

Histological type

Serous

Endometrioid

Undifferentiated

Clear cell

Transitional

Carcinosarcoma

Debulking surgery

Complete or optimal

Suboptimal

Type of surgery

With staging

Without staging

Chemotherapy

Paclitaxel + carboplatin

Docetaxel + carboplatin

Ifosfamide + epirubicin + cisplatin

Paclitaxel alone

None
No. of patients

60

8

$60(38-85)$

64

4

14

54

27

21

5

2

10

3

$338.5(4-16,000)$

45

9

9

2

3

10

58

11

8

40

9

50

6

2

1

1

8

44

24

34

34 
Table II. Patient outcome.

\begin{tabular}{lc}
\hline Outcome & No. of patients \\
\hline Response evaluation & 56 \\
Complete response & 10 \\
Partial response & 1 \\
Stable disease & 1 \\
Progressive disease & \\
Recurrence & 34 \\
Yes & 34 \\
No & \\
Secondary debulking surgery & $(44.1 \%)$ \\
Yes & 19 \\
No & 17.5 \\
Progression-free survival (median, months) & $77.2 \%$ \\
Three-year survival rate & \\
\hline
\end{tabular}

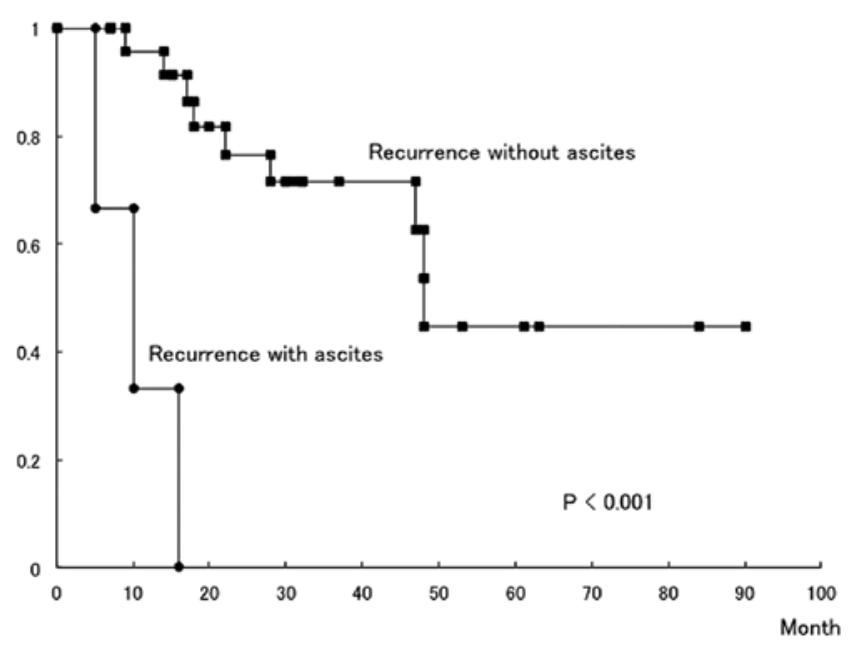

Figure 1. Survival time after recurrence. The median survival time after recurrence was 28 months: 7.5 and 30 months with and without ascites, respectively $(\mathrm{P}<0.001)$.

was 60 years (range, 38-85 years); initial symptoms included so-called Latzko's triad of lower abdominal pain (including bloating) in 27 patients (39.7\%), atypical genital bleeding in 21 patients $(30.9 \%)$, and the sensation of an abdominal mass in 5 patients $(7.4 \%)$. Melena due to infiltration of the bowel was the initial symptom in 2 patients. Preoperative diagnoses included a suspected ovarian carcinoma in 45 patients $(66.2 \%)$; fallopian tube carcinoma was suspected before surgery in just 9 patients (13.2\%). Endometrial carcinoma was suspected and treatment for this condition was started in 9 patients $(13.2 \%)$. Histological types were serous adenocarcinoma in 50 patients, endometrioid adenocarcinoma in 6 patients, and other types in 4 patients. All of the 8 carcinosarcomas were heterologous. The clinical stages were: stage I in 11 cases, stage II in 8 cases, stage III in 40 cases, and stage IV in 9 cases, indicating that
Table III. Sites of recurrence.

\begin{tabular}{lc} 
Recurrent site $^{\mathrm{a}}$ & No. of patients $(\%)$ \\
\hline Intraperitoneal (with ascites) & $4(9.5)$ \\
Intrapertitoneal (without ascites) & $8(19.0)$ \\
Pelvic cavity & $11(26.2)$ \\
Lymph nodes & $8(19.0)$ \\
Liver & $7(16.7)$ \\
Lung & $3(7.1)$ \\
Increase in CA-125 alone & $1(2.5)$
\end{tabular}

Included are 30 recurrent carcinomas and 4 recurrent carcinosarcomas of the fallopian tube. Several relapsed patients had one or more recurrent sites.

stage III or more comprised $72 \%$ of the cases. All of the 68 patients underwent standard surgery, and additionally staging surgery was performed in 34 patients. Optimal surgery was achieved in 44 patients (64.7\%) (including 10 patients who underwent associated intestinal resection) and suboptimal surgery was achieved in 24 patients. The median number of chemotherapy cycles was 6 (range, 1-12). Postoperative chemotherapy included platinum-based combination treatment in 64 patients $(94 \%)$.

Patient outcome. Patient outcome is summarized in Table II. The initial therapeutic effect in 68 patients with this disease was $\mathrm{CR}$ in 56 patients and $\mathrm{PR}$ in 10 patients, indicating that a response rate as high as $97.1 \%$ was achieved. The median observation period for FTM was 41 months (8-126 months). Three of 19 patients with stage I/II disease experienced recurrence and one patient died. The patient who died was a patient who received incomplete chemotherapy due to the presence of chronic renal failure. Recurrence occurred in 31 of 49 stage II/IV patients (63\%), with a median PFS of 17.5 months, and a 3 -year OS of $77.2 \%$.

While the most frequent site of recurrence was intraperitoneal dissemination, local pelvic peritoneal recurrence (26.2\%) and solitary recurrences in the lymph nodes $(19.0 \%)$ and the liver (16.7\%) were relatively frequent (Table III). Secondary debulking surgery (SDS) such as resection of the relapsed abdominal/pelvic mass or lymph nodes, or partial hepatectomy was performed in as many as $44 \%$ of patients with recurrence (Table II). Conversely, recurrence was associated with ascites (carcinomatous peritonitis) in just 4 of the relapsed 34 patients, but all 4 patients died. The median survival time after recurrence was 28 months: 7.5 and 30 months with and without ascites, respectively $(\mathrm{P}<0.001)$ (Fig. 1).

Determination of factors predicting poor outcome in fallopian tube malignancies. Univariate analysis by histological type, FIGO stage, age, CA-125 level, operative procedure, completion rate, recurrent site, presence or absence of SDS performance, and time to recurrence was performed to assess predictive factors related to the prognosis of FTM. Prognosis was significantly correlated solely with whether SDS could be performed (Table IV). 
Table IV. Determination of factors predicting poor outcome in fallopian tube malignancy.

\begin{tabular}{lll}
\hline Factors & No. of patients $\quad$ Death events from this disease P-value \\
\hline
\end{tabular}

Histology

Carcinoma of the fallopian tube

60

8

Carcinosarcoma of the fallopian tube

Stage

I/II

III/IV

49

33

35

$<60$

CA-125 value

$\geq 330$

$<330$

44

24

Complete or optimal

Suboptimal

Staging laparotomy

Yes

No

Intraperitoneal

Others

Ascites in recurrence

Yes

Secondary debulking surgery

Yes

No

$$
30
$$

2
12

ns

2

13

1

ns, not significant.

\section{Discussion}

This study revealed that, although intraperitoneal recurrence occurred in fallopian tube carcinoma and carcinosarcoma, recurrence presented as carcinomatous peritonitis was noted in only 4 of the 34 patients with relapsed disease; isolated metastases to the abdominal/pelvic cavity or lymph nodes and sole metastasis to the liver were relatively frequent. Therefore, it appears that post-recurrence survival was prolonged since in many patients SDS such as excision of the relapsed abdominal/ pelvic mass or the relapsed lymph node or partial hepatic resection was indicated. This fact was also supported by the univariate analysis which revealed that prognosis was significantly correlated solely with whether SDS was performed. Previous studies have shown that FTM easily infiltrates surrounding organs and progresses aggressively (1), and that lymph node metastasis occurs more frequently than that in ovarian carcinoma (9). The present study, however, showed that only 4 of the 34 patients with recurrent disease developed carcinomatous peritonitis, demonstrating for the first time that carcinomatous peritonitis is uncommon. It is already known in cases of ovarian carcinoma that the prognosis of a solitary recurrence in the liver is significantly improved by partial hepatic resection (10). Thus, the results of the present study suggest that SDS should be positively introduced to recurrent FTMs unless patients develop carcinomatous peritonitis.

With regard to the initial site of recurrence, FTM shows contrasting findings with those for ovarian carcinoma (Table III). While the present results demonstrated a high frequency of lymph nodes recurrence $(19.0 \%)$ and solitary liver metastasis (16.7\%), Ushijima reported that lymph node metastases $(7.1 \%)$ and solitary liver metastases $(6.3 \%)$ occur 
infrequently in ovarian carcinoma (11). FTM is richly permeated with lymphatic channels that drain into the para-aortic lymph nodes through infundibulopelvic lymphatics (12), and indeed a more frequent rate of lymph node metastasis than ovarian carcinoma has been reported $(9,11)$. In addition, Ajithkumar et al (13) found that metastasis to the retroperitoneal lymph nodes was observed at the initial visit in $\sim 50 \%$ of patients, irrespective of the stage of disease advancement. In this study as well, metastasis to the retroperitoneal lymph nodes was observed in 16 of the 34 patients (47.1\%) on whom staging surgery was conducted initially (data not shown).

It has been reported that platinum-based chemotherapy for advanced or recurrent fallopian tube carcinoma resulted in CR in $75 \%$ of patients (14) and that an $82.2 \%$ response rate was obtained in progressive fallopian tube carcinoma (9). In this study, the combination therapy with platinum and a taxane was chosen as a postoperative chemotherapeutic regimen for most patients, and CR to postoperative chemotherapy was $82 \%$ in the present patients, although suboptimal surgery accounted for $\sim 35 \%$ of all patients. There was no difference in the number of deaths between patients who received optimal surgery and those who received suboptimal surgery, suggesting that aggressive postoperative chemotherapy was mandatory and the combination of platinum and a taxane was effective, even in patients undergoing suboptimal surgery. The results of the present study were compatible with those of Baekelandt et al (15) and Gemignani et al (16), in which an $87.5 \%$ response rate and significant prolongation of PFS were observed. These reports and this study suggest that combination therapy with platinum and a taxane is effective against FTM. Given that the response rate to chemotherapy in epithelial ovarian carcinoma was found to be $56 \%$ (17) and that the rate for ovarian carcinosarcoma was 20 to $68 \%(18,19)$, the favorable effect of initial therapy in FTM is noteworthy. This favorable therapeutic effect may be attributed to the histological type of fallopian tube carcinoma, which is largely composed of serous and endometrioid adenocarcinoma.

Within Latzko's triad, atypical genital bleeding was observed in $\sim 30 \%$ of patients in this study. If an adnexal tumor is detected in patients with atypical genital bleeding or a watery vaginal discharge, FTM should be considered as a differential diagnosis. Nevertheless, a definitive preoperative diagnosis of FTM is difficult in many patients. It has been reported that FTM is diagnosed preoperatively in 0 to $10 \%$ of patients (20-22). Moreover, Meng et al (23) reported that in $50 \%$ of patients, even intraoperative findings fail to identify that the primary site is the fallopian tube. In this study, preoperative diagnosis was feasible in $13.2 \%$ of patients, probably by virtue of advances in imaging diagnosis. Transvaginal ultrasonography has been reported to be more effective than abdominal ultrasonography (24), and the presence of a sausage-like mass and/or multilobular mass with a 'cog-and-wheel' appearance is considered to be the basis for suspecting a FTM (25-27). Diagnostic ability has improved with the development of color Doppler and three-dimensional Doppler transvaginal ultrasonography (28). However, findings that clearly discriminate FTM have not been identified, even with full use of CT and MRI. Although this provides no clinical specificity, the CA-125 level is increased in $80 \%$ or more of patients with fallopian tube carcinoma $(13,29)$. Although it has been reported occasionally that the preoperative CA-125 level is an independent predictive factor for the prognosis of fallopian tube carcinoma $(13,30)$ and that this level correlates with responsiveness to chemotherapy $(31,32)$, such a correlation with the prognosis and therapeutic effect was not observed in this study. More cases need to be accumulated to establish the clinical significance of the CA-125 level in fallopian tube carcinoma.

A retrospective multicenter study was conducted recently to clarify the prognosis of fallopian tube carcinoma. Moore et al (33) used matched, case-controlled methods to compare the prognosis of fallopian tube carcinoma in 96 patients and epithelial ovarian carcinoma in 189 patients. Comparison of stage I/II with a median observation period of 57 months for fallopian tube carcinoma and 42 months for ovarian carcinoma showed a 5-year survival rate of $95 \%$ for fallopian tube carcinoma and $76 \%$ for ovarian carcinoma $(\mathrm{P}=0.02)$; a similar comparison in stage III/IV disease with a median observation period of 33 months for fallopian tube carcinoma and 35 months for ovarian carcinoma, showed a 3-year survival rate that was comparable (59\%) for both diseases. Accordingly, Moore et al (33) re-evaluated the concept that therapy for fallopian tube carcinoma could be similar to that for ovarian carcinoma. Conversely, Pectasides et al (34) analyzed 64 patients with fallopian tube carcinoma and reported that advanced stage and residual tumor diameter correlated significantly with prognosis. In stage III/IV fallopian tube carcinoma, while the 5-year survival in patients whose residual tumors could be debulked to less than $1 \mathrm{~cm}$ was $55 \%$, the 5-year survival of patients with larger residual tumor deposits was $21 \%$ (9). Thus, optimal debulking and subsequent platinum/taxan therapy were concluded to confer a favorable prognosis. In addition, a large population-based tumor registry study in which 416 patients with fallopian tube carcinoma were included showed that the 5-year survival rate was $95 \%$ for stage I, 75\% for stage II, $69 \%$ for stage III, and $45 \%$ for stage IV, respectively, indicating that the prognosis was better than the 5-year survival rate for each stage of ovarian carcinoma evaluated at around the same time (35). More recently, using SEER database, the outcome of 1,576 women with fallopian tube carcinoma and 54,249 with epithelial ovarian carcinoma was compared (4). Five-year cancer-specific survival was 54 and $36 \%$ in women with stage III and IV fallopian tube carcinoma, respectively, vs. only 30 and $14 \%$ in those with the same stage epithelial ovarian cancer, respectively (4). However, initially relapsed locations and time to recurrence were not clarified in the SEER database (4). The present results suggest that there are many isolated recurrences and few recurrences of carcinomatous peritonitis for FTMs. Thus, because there are many cases of FTMs for which recurrent tumors can be extracted by surgery, even if the disease relapsed, prognosis would be improved. Although a hypothesis for the origin of ovarian and peritoneal carcinoma has been proposed, based on the concept of transport and implantation of malignant cells from the fallopian tube to the ovary and peritoneum (36), the results of this study suggest that FTM has a different biological behavior when compared to ovarian carcinoma.

The present study suggests that FTM frequently results in solitary recurrence, and that aggressive recurrence treatment including SDS would improve the prognosis of this disease. 
However, several limitations must be acknowledged in this study. While differentiation of fallopian tube and ovarian carcinoma for women with advanced stage disease is sometimes difficult (37), a central pathology review was not performed as a retrospective nature. Although clinical features of fallopian tube carcinoma are not distinct from those of ovarian carcinoma as reported using case-control analysis (38), this study did not adopt a case-control method. Further study to elucidate the molecular mechanisms involved in the development of FTM and to improve decision-making for disease treatment is warranted.

\section{References}

1. Pectasides D, Pectasides E and Economopoulos T: Fallopian tube carcinoma: a review. Oncologist 11: 902-912, 2006.

2. Rosenblatt KA, Weiss NS and Schwartz SM: Incidence of malignant fallopian tube tumors. Gynecol Oncol 35: 236-239, 1989.

3. Riska A, Leminen A and Pukkala E: Sociodemographic determinations of incidence of primary fallopian tube carcinoma, Finland 1953-97. Int J Cancer 104: 643-645, 2003.

4. Wethington SL, Herzog TJ, Seshan VE, et al: Improved survival for fallopian tube cancer: a comparison of clinical characteristics and outcome for primary fallopian tube and ovarian cancer. Cancer 113: 3298-3306, 2008.

5. Sedlis A: Primary carcinoma of the fallopian tube. Obstet Gynecol Surv 16: 209-226, 1961.

6. World Health Organization Classification of Tumours: Pathology \& Genetics. Tumours of the Breast and Female Genital Organs. IARC Press, Lyon, 2003.

7. Hoskins WJ, McGuire WP, Brady MF, et al: The effect of diameter of largest residual disease on survival after primary cytoreductive surgery in patients with suboptimal residual epithelial ovarian carcinoma. Am J Obstet Gynecol 170: 974-979, 1994.

8. Eisenhauer EA, Therasse P, Bogaerts J, et al: New response evaluation criteria in solid tumours: revised RECIST guideline (version 1.1). Eur J Cancer 45: 228-247, 2009.

9. Gadducci A, Landoni F, Sartori E, et al: Analysis of treatment failures and survival of patients with fallopian tube carcinoma: a Cooperation Task Force (CTF) study. Gynecol Oncol 81: 150-159, 2001

10. Roh HJ, Kim DY, Joo WD, et al: Hepatic resection as part of secondary cytoreductive surgery for recurrent ovarian cancer involving the liver. Arch Gynecol Obstet 284: 1223-1229, 2011.

11. Ushijima K: Treatment of recurrent ovarian cancer - at first relapse. J Oncol 2010: 497429, 2010.

12. Klein M, Rosen AC, Lahousen M, et al: Lymphadenectomy in primary carcinoma of the fallopian tube. Cancer Lett 147: 63-66, 1999.

13. Ajithkumar TV, Minimole AL, John MM and Ashokkumar OS Primary fallopian tube carcinoma. Obstet Gynecol Surv 60 247-252, 2005.

14. Peter WA III, Andersen WA, Hopkins MP, et al: Prognostic features of carcinoma of the fallopian tube. Obstet Gynecol 71: 757-762, 1988

15. Baekelandt M, Joruun Nesbakken A, Kristensen GB, et al: Carcinoma of the fallopian tube. Cancer 89: 2076-2084, 2000.

16. Gemignani ML, Hensley ML, Cohen R, et al: Paclitaxel-based chemotherapy in carcinoma of the fallopian tube. Gynecol Oncol 80: 16-20, 2001.
17. Katsumata N, Yasuda M, Takahashi F, et al: Dose-dense paclitaxel once a week in combination with carboplatin every 3 weeks for advanced ovarian cancer: a phase 3, open-label, randomised controlled trial. Lancet 374: 1331-1338, 2009.

18. Anderson B, Turner DA and Benda J: Ovarian sarcoma. Gynecol Oncol 26: 183-192, 1987.

19. Tate Thigpen J, Blessing JA, DeGeest K, et al: Cisplatin as initial chemotherapy in ovarian carcinosarcima: a Gynecologic Oncology Group study. Gynecol Oncol 93: 336-339, 2004

20. Eddy GL, Copeland LJ and Gershenson DM: Second-look laparotomy in fallopian tube carcinoma. Gynecol Oncol 19: 182-186, 1984.

21. Podratz KC, Podczaski ES, Gaffey TA, et al: Primary carcinoma of the fallopian tube. Am J Obstet Gynecol 154: 1319-1326, 1986.

22. Huber-Buchholz MM, Buchholz NP and Staehelin J: Analysis of 23 cases of primary carcinoma of the fallopian tube over 50 years. J Obstet Gynaecol Res 22: 193-199, 1996.

23. Meng ML, Gan-Gao, Scheng-Sun, et al: Diagnosis of primary adenocarcinoma of the fallopian tube. J Cancer Res Clin Oncol 110: 136-140, 1985.

24. Timor-Tritsch IE and Rottem S: Transvaginal ultrasonographic study of the fallopian tube. Obstet Gynecol 70: 424-428, 1987.

25. Subramanyam BR, Raghavendra BN, Whalen CA, et al: Ultrasonic features of fallopian tube carcinoma. J Ultrasound Med 3: 391-393, 1984.

26. Kol S, Gal D, Friedman M and Paldi E: Preoperative diagnosis of fallopian tube carcinoma by transvaginal sonography and CA-125. Gynecol Oncol 37: 129-131, 1990.

27. Ajjimakorn S, Bhamarapravati Y and Israngura N: Ultrasound appearance of fallopian tube carcinoma. J Clin Ultrasound 16 : 516-518, 1988.

28. Kurjak A, Kupesic S, Sparac V and Kosuta D: Three-dimensional ultrasonographic and power Doppler characterization of ovarian lesions. Ultrasound Obstet Gynecol 16: 365-371, 2000.

29. McMurray EH, Jacobs AJ, Perez CA, et al: Carcinoma of the fallopian tube. Management and sites of failure. Cancer 58: 2070-2075, 1986.

30. Rosen AC, Klein M, Hafner E, et al: Management and prognosis of primary fallopian tube carcinoma. Austrian Cooperative Study Group for Fallopian Tube Carcinoma. Gynecol Obstet Invest 47: 45-51, 1999.

31. Tokunaga T, Miyazaki K, Matsuyama S, et al: Serial measurement of CA 125 in patients with primary carcinoma of the fallopian tube. Gynecol Oncol 36: 335-337, 1990.

32. Hefler LA, Rosen AC, Graf AH, et al: The clinical value of serum concentrations of cancer antigen 125 in patients with primary fallopian tube carcinoma: a multicenter study. Cancer 89: $1555-1560,2000$

33. Moore KN, Moxley KM, Fader AN, et al: Serous fallopian tube carcinoma: a retrospective, multi-institutional case-control comparison to serous adenocarcinoma of the ovary. Gynecol Oncol 107: 398-403, 2007.

34. Pectasides D, Pectasides E, Papaxoinis G, et al: Primary fallopian tube carcinoma: results of a retrospective analysis of 64 patients. Gynecol Oncol 115: 97-101, 2009.

35. Kosary C and Trimble EL: Treatment and survival for women with fallopian tube carcinoma: a population-based study. Gynecol Oncol 86: 190-191, 2002.

36. Kurman RJ and Shih IeM: Molecular pathogenesis and extraovarian origin of epithelial ovarian cancer - shifting the paradigm. Hum Pathol 42: 918-931, 2011.

37. Berek JS, Crum C and Friedlander M: Cancer of the ovary, fallopian tube, and peritoneum. Int J Gynaecol Obstet 119: S118-S129, 2012

38. Dunn MS, Manahan KJ and Geisler JP: Primary carcinoma of the fallopian tube and epithelial ovarian carcinoma: a casecontrol analysis. J Reprod Med 53: 691-694, 2008. 